\title{
Liver parameters as part of a non-invasive model for prediction of all-cause mortality after myocardial infarction
}

Theodor Baars ${ }^{1}$, Jan-Peter Sowa ${ }^{2}$, Ursula Neumann ${ }^{3}$, Stefanie Hendricks ${ }^{1}$, Mona Jinawy ${ }^{1}$, Julia Kälsch², Guido Gerken², Tienush Rassaf ${ }^{1}$, Dominik Heider ${ }^{3,4}$, Ali Canbay²

\author{
1Department for Cardiology, West German Heart and Vascular Centre Essen, \\ University Hospital, University Duisburg-Essen, Essen, Germany \\ 2Department of Gastroenterology and Hepatology, University Hospital, \\ University Duisburg-Essen, Essen, Germany \\ ${ }^{3}$ Department of Bioinformatics, Straubing Center of Science, University of Applied \\ Science Weihenstephan-Triesdorf, Straubing, Germany \\ ${ }^{4}$ Department of Mathematics and Computer Science, University of Marburg, Marburg, \\ Germany
}

Submitted: 9 June 2017

Accepted: 29 June 2017

Arch Med Sci 2020; 16 (1): 71-80

DOI: https://doi.org/10.5114/aoms.2018.75678

Copyright $\odot 2018$ Termedia \& Banach

\section{Abstract}

Introduction: Liver parameters are associated with cardiovascular disease risk and severity of stenosis. It is unclear whether liver parameters could predict the long-term outcome of patients after acute myocardial infarction (AMI). We performed an unbiased analysis of the predictive value of serum parameters for long-term prognosis after AMI.

Material and methods: In a retrospective, observational, single-center, cohort study, 569 patients after AMI were enrolled and followed up until 6 years for major adverse cardiovascular events, including cardiac death. Patients were classified into non-survivors $(n=156)$ and survivors $(n=413)$. Demographic and laboratory data were analyzed using ensemble feature selection (EFS) and logistic regression. Correlations were performed for serum parameters.

Results: Age (73; 64; $p<0.01)$, alanine aminotransferase (ALT; $93 \mathrm{U} / \mathrm{l}$; $40 \mathrm{U} / \mathrm{l} ; p<0.01$ ), aspartate aminotransferase (AST; $162 \mathrm{U} / \mathrm{l} ; 66 \mathrm{U} / \mathrm{l} ; p<0.01$ ), C-reactive protein (CRP; $4.7 \mathrm{U} / \mathrm{l} ; 1.6 \mathrm{U} / \mathrm{l} ; p<0.01)$, creatinine $(1.6 ; 1.3$; $p<0.01$ ), $\gamma$-glutamyltransferase (GGT; $71 \mathrm{U} / \mathrm{l} ; 46 \mathrm{U} / \mathrm{l} ; p<0.01)$, urea (29.5; $20.5 ; p<0.01$ ), estimated glomerular filtration rate (eGFR; 49.6; 61.4; $p<0.01)$, troponin $(13.3 ; 7.6 ; p<0.01)$, myoglobin $(639 ; 302 ; p<0.01)$, and cardiovascular risk factors (hypercholesterolemia $p<0.02$, family history $p<0.01$, and smoking $p<0.01$ ) differed significantly between non-survivors and survivors. Age, AST, CRP, eGFR, myoglobin, sodium, urea, creatinine, and troponin correlated significantly with death $(r=-0.29 ; 0.14 ; 0.31 ;-0.27$; $0.20 ;-0.13 ; 0.33 ; 0.24 ; 0.12)$. A prediction model was built including age, CRP, eGFR, myoglobin, and urea, achieving an AUROC of $77.6 \%$ to predict long-term survival after AMI.

Conclusions: Non-invasive parameters, including liver and renal markers, can predict long-term outcome of patients after AMI.

Key words: non-invasive prediction, liver enzymes, percutaneous coronary intervention, troponin.
Corresponding author: Prof. Ali Canbay MD Department of Gastroenterology and Hepatology University Hospital University Duisburg-Essen Leipzigerstr. 44 39120 Magdeburg Essen, Germany Phone: +49 3916713100 E-mail: ali.canbay@med. ovgu.de 


\section{Introduction}

The liver is the central organ in glucose, lipid, protein, and vitamin metabolism and produces coagulation factors and serum proteins (albumin, C-reactive protein (CRP)) [1]. Based on this variety of important functions for the whole organism, liver damage may affect other organ systems. The most prominent and most common example of this is non-alcoholic fatty liver disease (NAFLD), which is the liver manifestation of metabolic syndrome (MS) [2]. Non-alcoholic fatty liver disease is caused by excess lipid accumulation, which may harm the liver without apparent serological signs of injury [3]. Non-alcoholic fatty liver disease represents an emerging public health problem worldwide and is a major cause of liver-related morbidity and mortality $[4,5]$. Non-alcoholic fatty liver disease is considered a multisystem disease [6], backed up by the fact that the most common cause of mortality in NAFLD is cardiovascular mortality [7, 8]. Evidence has accumulated suggesting a strong association between NAFLD and cardiovascular disease (CVD), for example different manifestations of subclinical atherosclerosis such as increased carotid intima-media thickness and carotid atherosclerosis [9, 10], endothelial dysfunction [11], arterial stiffness [12], impaired left ventricular function [13], and coronary calcification $[9,14]$. In addition, increased serum levels of common markers of liver injury ( $\gamma$-glutamyltransferase (GGT), alanine aminotransferase (ALT), aspartate aminotransferase (AST), alkaline phosphatase (AP), and bilirubin) are associated with greater risk of CVD events [15-18]. Recently, we demonstrated a link between liver enzymes and the severity of stenosis in acute myocardial infarction (AMI) [19]. Serum liver markers were relevant factors to identify high-risk subjects in a non-invasive prognostic score. There is also emerging evidence that NAFLD is associated with increased prevalence and incidence of chronic kidney disease (CKD), independently of multiple cardio-renal risk factors [20, 21].

The prognosis for patients after an AMI depends on several factors. Known factors include the extent of myocardium undergoing irreversible injury [22] and the type of the AMI (ST-elevation myocardial infarction (STEMI) versus non-ST-elevation myocardial infarction (NSTEMI)) [23], the time point of reperfusion [24], and the type of the implanted stent [23]. Age [25], gender [26], and cardiovascular risk factors are further essential factors which affect AMI patients' outcome. However, it is currently unknown whether liver injury in early stages represented by liver enzymes is also associated with the long-term prognosis of patients after $\mathrm{AMI}$ or whether other non-invasive factors may have predictive properties.
In this retrospective study, data of patients after $\mathrm{AMI}$ were collected and analyzed for the potential predictive value of unbiased non-invasive parameters, including liver serum markers, for the long-term prognosis of patients after AMI. Demographic data and classic serum parameters for heart, renal, and liver diseases were analyzed for correlations with death.

\section{Material and methods}

\section{Ethics statement}

The study protocol conformed to the ethical guidelines of the 1975 Declaration of Helsinki and was approved by the Institutional Review Board (IRB; Ethik-Kommission der Medizinischen Fakultät der Universität Duisburg-Essen; Germany; reference number 15-6356-BO). Due to the retrospective nature of the study the IRB waived the need for written informed consent. All procedures adhered to the Declaration of Helsinki and the requirements of the IRB.

\section{Study design and sample acquisition}

In a retrospective single-center study, a cohort of 1242 patients was recruited. All patients had an $\mathrm{AMI}$ and underwent coronary angiography in the catheterization laboratory of the West German Heart and Vascular Centre Essen, University Hospital Essen. Acute myocardial infarction was defined as a troponin value above the $99^{\text {th }}$ percentile of the upper reference level and either with ST-segment elevation or new left bundle-branch block on the electrocardiogram (STEMI, $n=117$ ) [27], or without ST-segment elevation or new left bundle-branch block on the electrocardiogram (NSTEMI, $n=452$ ) [28]. Patients were classified in two groups, non-survivors $(n=156)$ and survivors ( $n=413)$, according to the follow-up of up to 6 years. Blood sampling was done directly before coronary intervention and serum parameters were determined in the central laboratory unit of the University Hospital Essen (Department of Clinical Chemistry and Laboratory Medicine) by standardized methods. Exclusion criteria were high-grade aortic valve disease, cardiomyopathy, cardiac allograft vasculopathy, endocarditis, hypertensive emergency, myocarditis, pericarditis, tachyarrhythmia absoluta by atrial fibrillation (atrial fibrillation $>100 \mathrm{bpm} / \mathrm{min}$ ), and survival of sudden cardiac death. In addition, patients with a coronary artery disease after saphenous vein aortocoronary bypass grafting (SVG surgery) were excluded from the study. Patients with non-cardiac causes of troponin elevation were excluded: acute neurological disease including stroke or cerebral hemorrhage, acute pulmonary embolism, aortic dissection, diseases such as amyloidosis, sarcoidosis, or sclero- 
derma, inflammatory myopathies such as polymyositis or dermatomyositis, sepsis, and patients who took cardio-toxic drugs (doxorubicin, 5-fluorouracil, trastuzumab). Finally, all patients for whom no follow-up was available were excluded (Figure 1, Flow diagram for patient selection), resulting in 569 eligible patients enrolled in the study.

\section{Outcome, definitions and clinical follow-up}

The main outcome of the study was cardiac death after 6 years. Secondary outcomes were major adverse cardiac events (MACE) at 6 years after the index procedure. The MACE was defined as one of the following conditions: AMI, cardiac death, unstable angina (UA), any repeat intervention inside the stent that had been implanted during the index procedure or within another segment or coronary vessel, stroke, and SVG surgery. Follow-up data were collected by telephone contact with the patient. No detailed workup for liver diseases was performed due to the emergency situation of AMI.

\section{Dataset and statistics}

The dataset (569 patients) included the socio-demographic parameters sex, age, and body mass index (BMI), as well as the serum parameters ALT, AST, bilirubin, CRP, creatinine, estimated glomerular filtration rate (eGFR), GGT, myoglobin, troponin, urea, potassium, and sodium.

\section{Correlation analysis}

Statistical data analyses were performed with $R$ (http://www.r-project.org/). All data are presented as mean \pm standard error of the mean (SEM) unless specified otherwise. Correlation analysis was performed using Spearman's rank correlation coefficient $r$.

\section{Importance analysis}

For the importance analysis and building a predictive model, missing values were deleted. The remaining sample size was $n=406$. The parameters were selected according to their importance obtained by an ensemble feature selection (EFS) approach. The EFS was calculated using the EFS-Server at http://efs.heiderlab.de. It provides an aggregation of eight feature selection methods, namely median, Pearson, and Spearman correlation, logistic regression, and four variable importance measures of the random forest algorithm [29]. We used the EFS server with default settings.

\section{Predictive modeling}

Predictive models were built up by a logistic regression as these models are easy to interpret and
Patients with a troponin value above the $99^{\text {th }}$ percentile of the upper reference level (January 2009-June 2014) $(n=1242)$

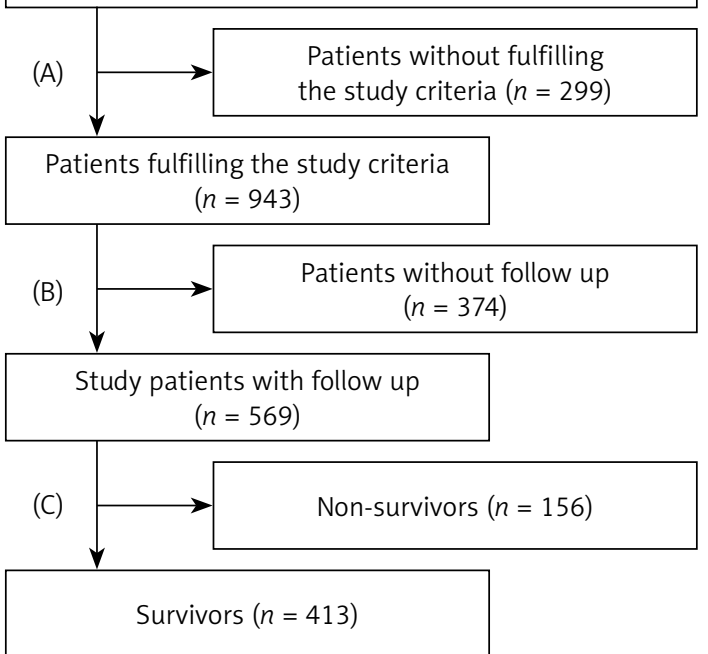

Figure 1. Recruitment and exclusion flow of patient cohort. Initially data of 1242 patients were screened retrospectively. All patients not fulfilling study criteria or without available follow-up were excluded. The remaining 569 patients were grouped according to survival

have been widely applied [30]. We used the implementation in the stats package of R (http://www.r-project.org) with standard settings. For evaluation of the classifier performance we followed the scheme of Sowa et al. [31]. A leave-one-out cross-validation scheme was used and the receiver operating characteristics (ROC) curve and the corresponding area under the curve (AUC) were calculated with pROC [32]. The $95 \% \mathrm{Cl}$ was computed with 2000 stratified bootstrap replicates. The ROC curve was built by plotting the sensitivity against the specificity for every possible cut-off between the two classes.

\section{Results}

\section{Patient characteristics, basic parameters, and clinical outcome}

Detailed data of the included patients grouped by survival can be found in Table I, comprising the distribution of socio-demographic parameters as well as serum parameters of heart, renal, and liver damage. Non-survivors were older and had significantly higher troponin, myoglobin, creatinine, urea, CRP, GGT, ALT, and AST levels. Non-survivors had significantly lower eGFR and prevalence of cardiovascular risk factors. Body mass index, bilirubin, and prevalence of STEMI or NSTEMI did not differ between the groups. Clinical follow-up was completed in 569 patients at 6 years (BMI: $27.5 \pm 0.2 \mathrm{~kg} / \mathrm{m}^{2}$; age: $66.3 \pm 0.6$; male/female: $406(71.3 \%) / 163(28.7 \%))$. Figure 2 depicts the 
T. Baars, J.P. Sowa, U. Neumann, S. Hendricks, M. Jinawy, J. Kälsch, G. Gerken, T. Rassaf, D. Heider, A. Canbay

Table I. Demographic and basic parameters of the patient cohort at enrollment

\begin{tabular}{|c|c|c|c|}
\hline Parameters & $\begin{array}{l}\text { Non-survivors } \\
\text { (total } n=156 \text { ) }\end{array}$ & $\begin{array}{c}\begin{array}{c}\text { Survivors } \\
\text { (total } n=413 \text { ) }\end{array} \\
\end{array}$ & $P$-value \\
\hline Age $(n=114$ vs. 381$)$ & $72.87 \pm 0.87$ & $64.37 \pm 0.65$ & $<0.01$ \\
\hline Gender ( $n$; female/male) & $53 / 103$ & $110 / 303$ & 0.096 \\
\hline $\mathrm{BMI}(n=97$ vs. 322$)$ & $27.03 \pm 0.55$ & $27.60 \pm 0.26$ & 0.32 \\
\hline GGT ( $n=156$ vs. 411$)$ & $70.54 \pm 9.0$ & $46.20 \pm 2.50$ & $<0.01$ \\
\hline AST $(n=155$ vs. 413$)$ & $161.63 \pm 41.72$ & $66.05 \pm 5.64$ & $<0.01$ \\
\hline $\operatorname{ALT}(n=156$ vs. 413$)$ & $93.07 \pm 24.43$ & $39.50 \pm 3.14$ & $<0.01$ \\
\hline Bilirubin ( $n=152$ vs. 385 ) & $0.65 \pm 0.03$ & $0.65 \pm 0.02$ & 0.95 \\
\hline $\operatorname{CRP}(n=152$ vs. 401$)$ & $4.72 \pm 0.51$ & $1.58 \pm 0.14$ & $<0.01$ \\
\hline Creatinine ( $n=154$ vs. 407$)$ & $1.64 \pm 0.08$ & $1.33 \pm 0.04$ & $<0.01$ \\
\hline Urea $(n=154$ vs. 406$)$ & $29.48 \pm 1.34$ & $20.47 \pm 0.74$ & $<0.01$ \\
\hline eGFR $(n=113$ vs. 378$)$ & $49.60 \pm 1.87$ & $61.41 \pm 0.91$ & $<0.01$ \\
\hline Troponin ( $n=155$ vs. 408$)$ & $13.28 \pm 2.32$ & $7.58 \pm 0.98$ & $<0.01$ \\
\hline Myoglobin ( $n=146$ vs. 381 ) & $639.29 \pm 132.78$ & $301.60 \pm 31.97$ & $<0.01$ \\
\hline STEMI & $27(26.7 \%)^{1}$ & $91(27.5 \%)^{1}$ & $1^{\mathrm{F}}$ \\
\hline NSTEMI & $74(73.3 \%)^{1}$ & $240(72.5 \%)^{1}$ & \\
\hline Ex-/smoker $(n=100$ vs. 324$)$ & $12 / 15(12 \% / 15 \%)$ & $80 / 92(24.7 \% / 28.4 \%)$ & $\chi^{2}:<0.01$ \\
\hline Type 2 diabetes & $28(27.7 \%$ of $n=101)$ & $108(33.4 \%$ of $n=323)$ & $0.3^{\mathrm{F}}$ \\
\hline Hypercholesterolemia & $73(73 \%$ of $n=100)$ & $272(83.6 \%$ of $n=325)$ & $0.0196^{\mathrm{F}}$ \\
\hline RR systolic ( $n=97$ vs. 324$)$ & 127.4 & 134.6 & 0.01 \\
\hline RR diastolic ( $n=97$ vs. 324 ) & 63.4 & 66.3 & 0.1 \\
\hline Heart rate $(n=80$ vs. 284$)$ & 80.9 & 75.9 & 0.06 \\
\hline Family history & $18(18.2 \%$ of $n=99)$ & $108(33.5 \%$ of $n=322)$ & $<0.01^{\mathrm{F}}$ \\
\hline
\end{tabular}

Continuous data are presented as mean \pm SEM, categorical data as absolute numbers. Comparison between survivors and non-survivors by Student's t test (continuous data) and Fisher's exact test $/ \chi^{2}$ (categorical data). ALT - alanine transaminase, AST-aspartate transaminase, $B M I$ - body mass index, CRP - C-reactive protein, eGFR - estimated glomerular filtration rate, GGT- $\gamma$-glutamyl transferase. ${ }^{1} P e r c e n t a g e s$ of 101 non-survivors and of 331 survivors with known STEMI/NSTEMI; Ftested with Fisher's exact test.

unadjusted Kaplan-Meier curves of events up to 6 years after $\mathrm{PCl}$. One hundred and fifty-six patients died during the follow-up period (Figure $2 \mathrm{~A}$ ). During follow-up 65 cases of UA occurred (known incidence for 416 patients; Figure 2 B), 61 cases of restenosis (known for 416) were observed (Figure 2 C), and 32 reinfarctions were recorded (of 419; Figure 2 D). Stroke occurred in 12 (of 414 known) cases and an SVG surgery had to be performed in 17 (of 417) cases.

Age and non-invasive parameters correlate significantly with death after myocardial infarction

Outcome defined as death after AMI was correlated with demographic and serum-derived parameters. Detailed findings of this analysis are presented in Table II. Higher age significantly correlated with death. Also routine cardiac parameters (troponin, myoglobin) correlated significantly with death. Additionally, serum parameters usually not used in cardiac diagnosis (AST, creatinine, CRP, eGFR, sodium, and urea) correlated with death. Other non-invasive parameters did not exhibit significant correlations (Table II). Liver parameters (ALT, AST) can be elevated in consequence of reduced arterial perfusion or congestion due to acute cardiac failure. To assess whether this may be the case in the present cohort, in a subgroup of 90 patients, in whom transthoracic echocardiography (TTE) was available, signs of right ventricular load (RVL) were evaluated. In 12 patients with TTE a sign of RVL was found and 2 of these exhibited NAFLD. In the 78 patients without signs of RVL, 3 patients had alcoholic liver disease, 5 had an unidentified liver disease, 
A

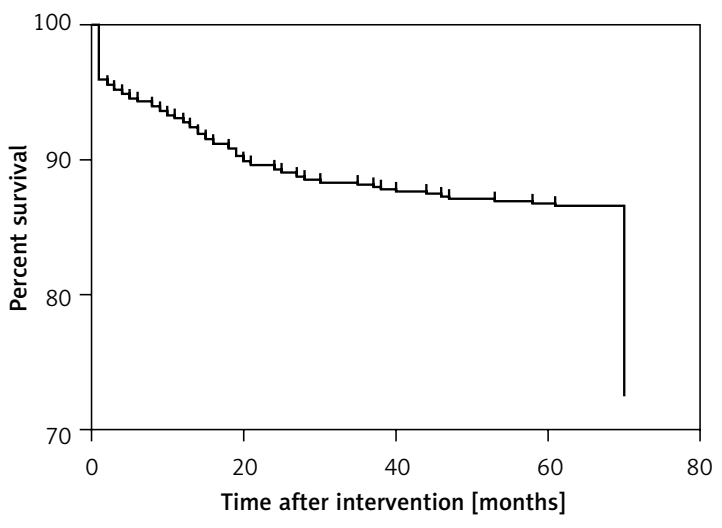

C

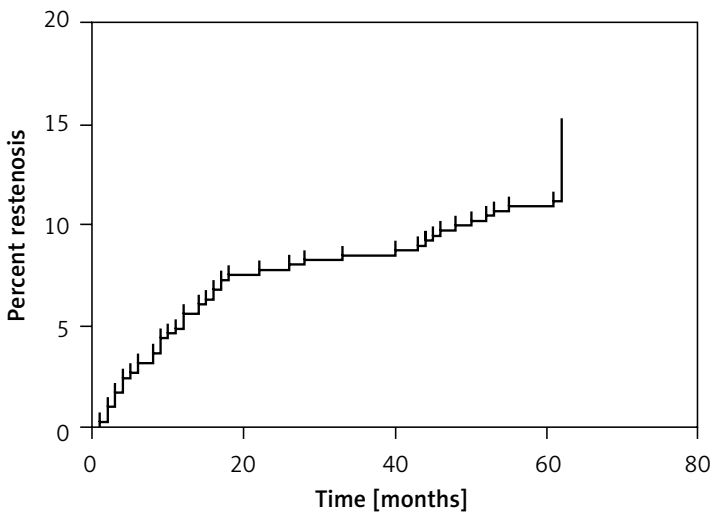

B

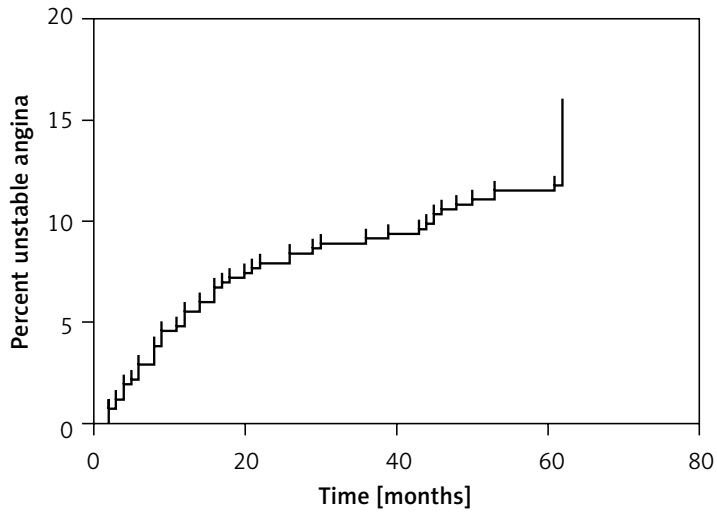

D

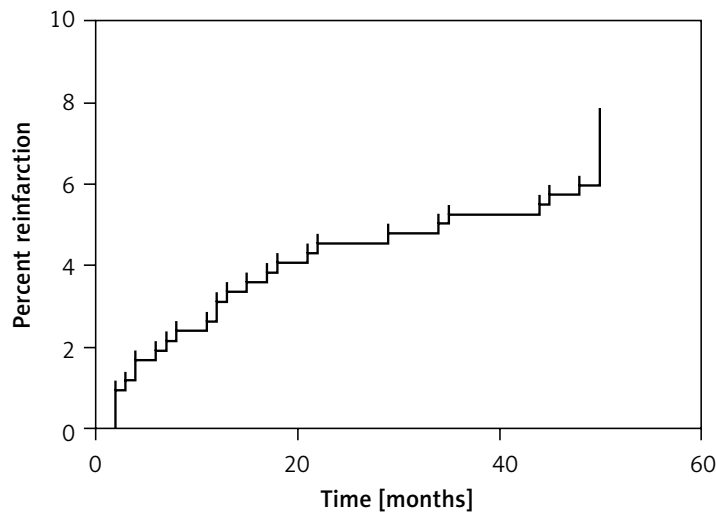

Figure 2. Unadjusted survival curves and cumulative incidence of MACE. Incidence of cardiac death (A) is given over a follow-up time of 70 months. Only individuals with a known outcome are depicted. Deceased patients for whom time of death was not known were censored at 70 months. Cumulative incidences of major adverse cardiac events (MACE) are depicted for unstable angina (UA; B), restenosis (C), and reinfarction (D). Only individuals with known outcome were included in this analysis (351 for UA and reinfarction; 350 for restenosis). Patients for whom time of the MACE occurrence was not known were censored at 62 months (UA, restenosis) or 50 months (reinfarction)

and a high proportion exhibited signs of metabolic alterations (Table III). While ALT and AST were nominally higher in patients with RVL, significance was not reached compared to patients without RVL (Table IV).

\section{Correlations of serum parameters among each other}

To assess whether non-invasive parameters for liver injury (ALT, AST, GGT) and routine heart parameters (troponin, myoglobin) were interrelated, correlation analysis was performed. All significant correlations are given in Table V. Both ALT and AST were correlated with myoglobin and troponin. In addition, GGT was inversely correlated with eGFR.

\section{High importance of ALT and vascular parameters for prediction of death}

To efficiently predict cardiac death after AMI an EFS approach was performed to select the most important non-invasive parameters for prediction.
Table II. Correlation of non-invasively determined parameters with death

\begin{tabular}{|lcc|}
\hline Parameter & $R^{1}$ & $P$-value \\
\hline Age & -0.2874 & $<0.0001$ \\
\hline ALT & -0.0199 & 0.6357 \\
\hline AST & 0.1377 & 0.001 \\
\hline Bilirubin & -0.0029 & 0.9470 \\
\hline Creatinine & 0.2384 & $<0.0001$ \\
\hline CRP & 0.3114 & $<0.0001$ \\
\hline eGFR & -0.268 & $<0.0001$ \\
\hline Myoglobin & 0.2035 & $<0.0001$ \\
\hline Sodium & -0.1313 & 0.0024 \\
\hline Troponin & 0.1167 & 0.0056 \\
\hline Urea & 0.3304 & $<0.0001$ \\
\hline
\end{tabular}

${ }^{1}$ Spearman's correlation coefficient $r$ of parameter $x$ with death. ALT - alanine transaminase, AST - aspartate transaminase, BMI - body mass index, CRP - C-reactive protein, eGFR - estimated glomerular fraction rate, GGT- $\gamma$-glutamyl transferase. 
T. Baars, J.P. Sowa, U. Neumann, S. Hendricks, M. Jinawy, J. Kälsch, G. Gerken, T. Rassaf, D. Heider, A. Canbay

Table III. Clinical observations in 100 patients with available transthoracic echocardiography

\begin{tabular}{|lcc|}
\hline Condition & $\begin{array}{c}\text { No signs of right ventricular load } \\
\text { present }(n=78)\end{array}$ & $\begin{array}{c}\text { Signs of right ventricular load } \\
\text { present }(n=12)\end{array}$ \\
\hline Alcoholic liver disease & 3 & 0 \\
\hline Non-alcoholic fatty liver disease & 0 & 2 \\
\hline Other liver disease & 5 & $9(75 \%)$ \\
\hline Hypercholesterolaemia & $63(83 \%$ of $n=76)$ & $5(42 \%)$ \\
\hline Obesity & $56(72 \%)$ & 0 \\
\hline
\end{tabular}

Table IV. Serum liver injury parameters in patients with available transthoracic echocardiography grouped by signs of right heart burden

\begin{tabular}{|lccc|}
\hline Parameter & $\begin{array}{c}\text { Signs of right ventricular load } \\
\text { present }(n=12)\end{array}$ & $\begin{array}{c}\text { No signs of right ventricular load } \\
\text { present }(n=78)\end{array}$ & $P$-value \\
\hline AST [U/I] & $316.3 \pm 62.1$ & $308.6 \pm 29.98$ & 0.92 \\
\hline ALT [U/I] & $230.7 \pm 142.1$ & $97.6 \pm 16.8$ & 0.37 \\
\hline
\end{tabular}

Table V. Correlations of serum derived parameters among each other

\begin{tabular}{|lccc|}
\hline Parameter 1 & Parameter 2 & $R$ & $P$-value \\
\hline AST & Troponin & 0.2743 & $<0.0001$ \\
\hline AST & Myoglobin & 0.1700 & $<0.0001$ \\
\hline AST & Urea & 0.1879 & $<0.0001$ \\
\hline ALT & Troponin & 0.2102 & $<0.0001$ \\
\hline ALT & Myoglobin & 0.2197 & $<0.0001$ \\
\hline ALT & Urea & 0.1804 & $<0.0001$ \\
\hline GGT & eGFR & -0.1409 & 0.0018 \\
\hline
\end{tabular}

$R$-Spearman's correlation coefficient of parameter. ALT - alanine transaminase, AST - aspartate transaminase, eGFR - estimated glomerular fraction rate, GGT - $\gamma$-glutamyl transferase.

Detailed importance measures for all included parameters are given in Figure 3. A high importance (above the average importance $=0.41$ ) could be identified for the variable age (0.76) as well as the serum parameters CRP (0.83), urea (0.73), eGFR (0.65), and myoglobin (0.49). The serum parameters ALT (0.4), creatinine (0.35), and sodium (0.37) reached reasonable importance (above [average $1 \times \mathrm{SEM}]=0.35$ ), while none of the other parameters exhibited a high importance and thus were excluded from further analyses.

\section{New diagnostic model for prediction of long-term death after acute myocardial infarction}

Based only on parameters with an EFS importance above average (age, CRP, eGFR, myoglobin, and urea), a prediction model was developed based on a logistic regression model. It achieved an AUC of $77.6 \%(95 \% \mathrm{Cl}: 72.5-82.6 \%)$ (Figure 4 A). Separating deceased patients with known

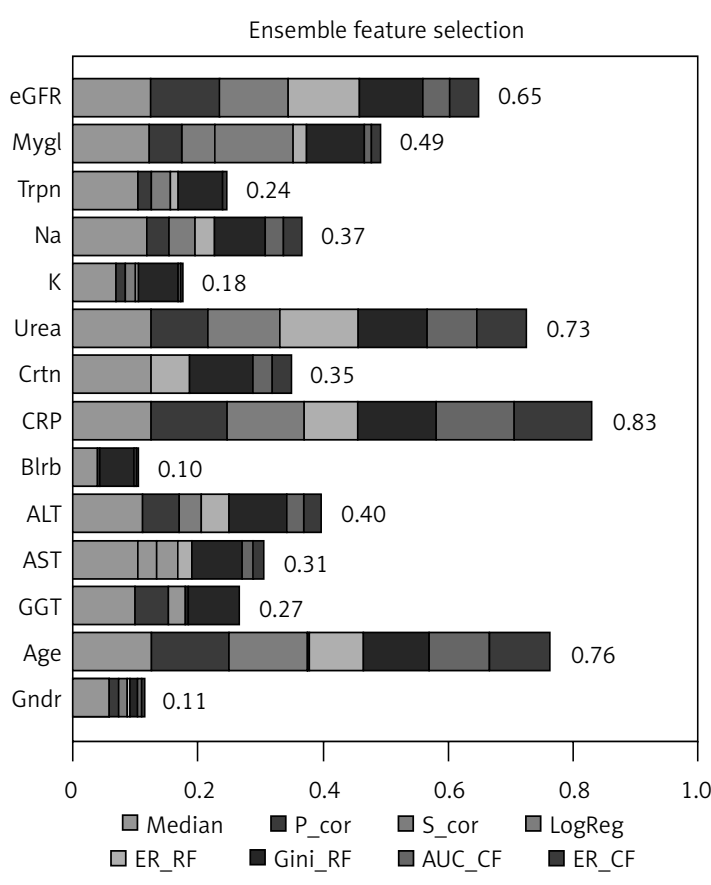

Figure 3. Ensemble feature selection importance analysis. The bars show the cumulative importance for the different parameters with respect to separation of death and survival. Higher numbers indicate greater importance for the classification. Partitions in the bars show contributions of single features of the ensemble selection approach to the total importance

P_cor - Pearson correlation, S_cor - Spearman correlation, LogReg - logistic regression, ER_RF - error rate of random forest, Gini RF - Gini impurity in random forest, AUC CF area under the curve for conditional random forest, $\bar{E} R_{-} C F$ - error rate of conditional random forest, eGFR - estimated glomerular filtration rate, Mygl-myoglobin, Trpn - troponin, Crtn - creatinine, CRP - C-reactive protein, Blrb - bilirubin, Gndr-gender.

time of death ( $n=76$ ) by low and high CRP (cutoff at $4.2 \mathrm{mg} / \mathrm{dl}$ ) resulted in significantly longer survival of patients with high CRP (Figure 4 B). 
A

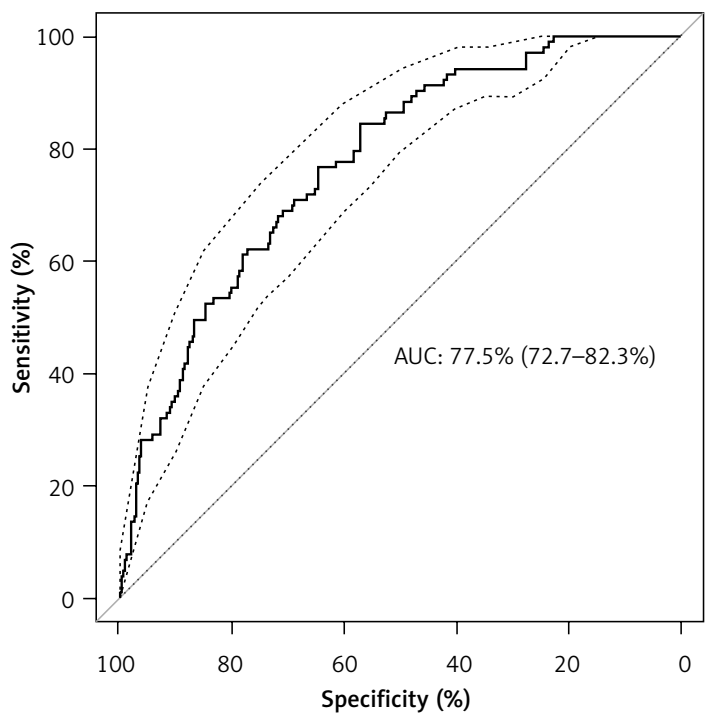

B

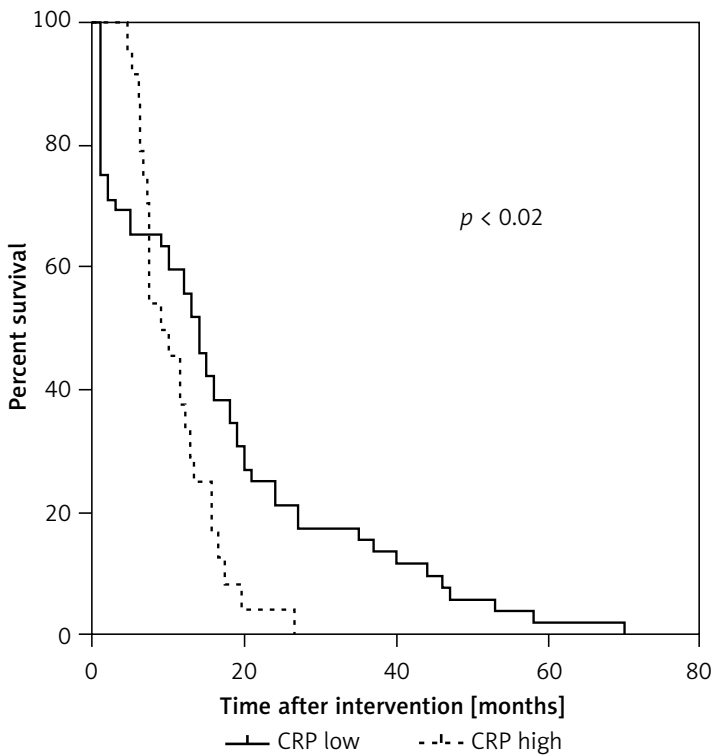

Figure 4. Performance of prediction model for prediction of cardiac death. (A) On the Y-axis the sensitivity and on the $\mathrm{X}$-axis the specificity is shown. The ROC curve is shown as a solid line. The AUC of the prediction model is $77.6 \%$ (95\% Cl: 72.5-82.6\%). The confidence interval is shown as a dashed line. The dotted line marks the performance of random guessing. (B) Kaplan-Meier analysis for C-reactive protein (CRP) as the most important parameter for prediction of death after acute myocardial infarction (AMI). Only patients with known time of death $(n=76)$ were included in the analysis and grouped according to low and high CRP (cutoff at $4.2 \mathrm{mg} / \mathrm{dl}$ ). Patients with high CRP had significantly shorter survival time after AMI

\section{Discussion}

In the present retrospective follow-up study, we have focused on patients after AMI and analyzed non-invasive markers for cardiac death. Using an EFS approach parameters with importance to predict long-term survival after AMI were identified and included not only classic cardiac markers, but also CRP, eGFR, and the classic liver parameter ALT. Additionally, liver parameters (ALT, AST and GGT) correlated significantly with heart parameters (myoglobin and troponin) and also with renal parameters (eGFR and urea). A prediction model was constructed with an AUC of $77.5 \%$ to predict cardiac death.

The parameters identified as important for non-invasive prediction of cardiac death corroborate previous findings of other groups. One of the most important factors identified was patient age, which is in line with the increased rate of mortality in elderly patients with AMI [33], and with a study of Dunn et al., comparing the survival of subjects with and without suspected NAFLD in a population-based cohort. Indeed, in this study, age was demonstrated to be a strong independent risk factor for cardiovascular death [34], similar to the cohort presented here. Age was the most important risk factor for in-hospital worsening of STEMI and distal embolization [35]. Also troponin was associated with cardiac death and relevant for prediction. In previous studies elevated troponin levels have been associated with a worse short- and long-term prognosis and the probability of death after AMI [36]. Another important factor for prediction of death after AMI was the eGFR. Again this is in line with previous findings, where renal impairment was associated with an increased risk of cardiac mortality $[25,37]$ and worse prognosis [38]. Serum creatinine and eGFR were identified as predictors of short- and long-term mortality in patients with AMI treated with primary angioplasty $[38,39]$. Generally, patients with CKD tend to be older and have more cardiovascular risk factors and higher incidence of MACE [40].

Another finding of our study was that routine liver parameters were significantly correlated with classic cardiac markers and eGFR. This indicates the relevance of liver-derived parameters for cardiac outcomes. The extent of liver injury, in particular in settings with metabolic syndrome and/or NAFLD, may have a direct impact on cardiac outcomes. Previous studies demonstrated that elevation of transaminases is a common phenomenon in patients within the acute phase of AMI [41] and that elevated transaminases are associated with MACE [42]. The admission levels of transaminases were associated with complications and mortality during intensive care stay in a large study with patients mechanically reperfused for AMI [43]. In our study only ALT showed a high importance to predict mortality in patients after AMI in a long follow-up period. This is in line with a study of Yun et al. demonstrating that elevated ALT levels are 
independently associated with increased CVD-related mortality in Koreans [44]. Indeed, ALT is very specific for liver injury and also for NAFLD [45]. Unfortunately, in the present cohort no detailed data on liver-related morbidity, viral hepatitides or other liver diseases were available. Nevertheless, routine liver parameters might be able to improve prediction of cardiac outcome, though larger studies are needed to confirm this hypothesis.

One possible explanation for the importance of liver- and kidney-related serum parameters for survival in AMI might be common underlying risk factors: insulin resistance, hyperlipidaemia, abdominal obesity and hypertension. Metabolic syndrome basically comprises these risk factors for cardiovascular disease, NAFLD and subsequent CKD. There is also accumulating evidence that NAFLD is an early mediator of MS as a systemic disease [46], and NAFLD has been associated with cardiovascular disease and CKD in many studies [9, 47-49]. Furthermore, advanced fibrosis in NAFLD predicted not only liver-related mortality [50] but also increased mortality due to cardiovascular events $[18,51,52]$. Severity of NAFLD seems to be paralleled by the reduction of eGFR and severity of CKD $[53,54]$. Although the exact underlying mechanism connecting elevated risk for CVD and CKD in NAFLD is still unknown, some data suggest a pivotal and causal role of NAFLD for more advanced metabolic injury, culminating in cardiovascular and renal outcomes [8, 20]. Apart from this, routine liver enzymes mostly exhibit no or only mild elevation in NAFLD $[55,56]$. This could be one reason for the relatively low relevance of serum liver parameters for prediction of cardiac mortality in this study. One limitation of the present study is that no detailed information on metabolic status or liver steatosis/NAFLD was present, as these are not routinely diagnosed in the emergency setting of AMI. This could be one explanation for the lower incidence of components of the metabolic syndrome in non-surviving patients. The proportion of individuals for whom this information was available differed between the groups. Another reason for this observation could be a more rigorous follow-up and faster response to any adverse events in patients with metabolic disturbances, leading to better survival. However, this is speculative and would need confirmation in prospective studies.

To predict mortality after AMI other scoring systems are in use, which mostly rely on parameters available during percutaneous intervention. These scores reach AUCS of up to $85 \%$ for prediction of 1 -year mortality $[39,57]$. In our study a scoring system comprising only non-invasive parameters reached an AUC of $77.6 \%$ for prediction of 6-year mortality. The best performance for commonly used scores for 6-year mortality (GRACE score) was also $77 \%$. It is interesting to note that diagnostic criteria of the metabolic syndrome and body fat distribution can predict the severity of an AMI, but not the outcome after the AMI [58]. One limitation of our study is that the time point of death was not known for all included patients. Another limitation is that the cause of death was not known in the majority of cases. Thus, the presented score should be interpreted as a prediction of overall mortality, independent of survival time (no separation of short- or long-term mortality or by cause of death). Due to the non-invasive nature of all used parameters, monitoring of cardiac health could be performed based on this or similar systems.

In conclusion, besides age, heart and renal parameters, liver parameters were associated with cardiac death and identified as possibly important for discrimination of non-survivors and survivors in a long-term follow-up of patients after AMI. On the one hand, cardiometabolic risk factors and common pathogenic mechanisms for CVD, CKD, and NAFLD should be analyzed for predictive properties and disease monitoring in this setting. On the other hand, a causal role for (an unknown) underlying liver disease should be considered to identify early markers of long-term cardiac outcomes.

\section{Acknowledgments}

This study was funded by the Dr. Heinz-Horst Deichmann-Stiftung.

\section{Conflict of interest}

The authors declare no conflict of interest.

\section{References}

1. Bechmann LP, Hannivoort RA, Gerken G, et al. The interaction of hepatic lipid and glucose metabolism in liver diseases. J Hepatol 2012; 56: 952-64.

2. Kim $\mathrm{CH}$, Younossi ZM Nonalcoholic fatty liver disease: a manifestation of the metabolic syndrome. Cleve Clin J Med 2008; 75: 721-8.

3. Kälsch J, Bechmann LP, Kälsch H, et al. Evaluation of biomarkers of NAFLD in a cohort of morbidly obese patients. J Nutr Metab 2011; 2011: 369168.

4. Fargion S, Porzio M, Fracanzani AL. Nonalcoholic fatty liver disease and vascular disease: state-of-the-art. World J Gastroenterol 2014; 20: 13306-24.

5. Younossi ZM, Koenig AB, Abdelatif D, et al. Global epidemiology of nonalcoholic fatty liver disease. Meta-analytic assessment of prevalence, incidence, and outcomes. Hepatology 2016; 64: 73-84.

6. Byrne CD, Targher G. NAFLD: a multisystem disease. J Hepatol 2015; 62 (1 Suppl): S47-64.

7. Siddiqui MS, Sterling RK, Luketic VA, et al. Association between high-normal levels of alanine aminotransferase and risk factors for atherogenesis. Gastroenterology 2013; 145: 1271-9.e1-3. 
8. Ahmed MH, Barakat S, Almobarak AO Nonalcoholic fatty liver disease and cardiovascular disease: has the time come for cardiologists to be hepatologists? J Obes 2012; 2012: 483135.

9. Oni ET, Agatston AS, Blaha MJ, et al. A systematic review: burden and severity of subclinical cardiovascular disease among those with nonalcoholic fatty liver: should we care? Atherosclerosis 2013; 230: 258-67.

10. Brea A, Puzo J. Non-alcoholic fatty liver disease and cardiovascular risk. Int J Cardiol 2013; 167: 1109-17.

11. Senturk O, Kocaman O, Hulagu S, et al. Endothelial dys function in Turkish patients with non-alcoholic fatty liver disease. Intern Med J 2008; 38: 183-9.

12. Ozturk K, Uygun A, Guler AK, et al. Nonalcoholic fatty liver disease is an independent risk factor for atherosclerosis in young adult men. Atherosclerosis 2015; 240: 380-6.

13. Bonapace S, Perseghin G, Molon G, et al. Nonalcoholic fatty liver disease is associated with left ventricular diastolic dysfunction in patients with type 2 diabetes. Diabetes Care 2012; 35: 389-95.

14. Kim D, Choi SY, Park EH, et al. Nonalcoholic fatty liver disease is associated with coronary artery calcification. Hepatology 2012; 56: 605-13.

15. Masoudkabir F, Karbalai S, Vasheghani-Farahani A, et al. The association of liver transaminase activity with presence and severity of premature coronary artery disease. Angiology 2011; 62: 614-9.

16. Mahabadi AA, Lehmann N, Möhlenkamp S, et al. Association of bilirubin with coronary artery calcification and cardiovascular events in the general population without known liver disease: the Heinz Nixdorf Recall study. Clin Res Cardiol 2014; 103: 647-53.

17. Schindhelm RK, Dekker JM, Nijpels G, et al. Alanine aminotransferase predicts coronary heart disease events: a 10-year follow-up of the Hoorn Study. Atherosclerosis 2007; 191: 391-6.

18. Targher G, Bertolini L, Rodella S, et al. Nonalcoholic fatty liver disease is independently associated with an increased incidence of cardiovascular events in type 2 diabetic patients. Diabetes Care 2007; 30: 2119-21.

19. Baars T, Neumann U, Jinawy $M$, et al. In acute myocardial infarction liver parameters are associated with stenosis diameter. Medicine 2016; 95: e2807.

20. Chang Y, Ryu S, Sung E, et al. Nonalcoholic fatty liver disease predicts chronic kidney disease in nonhypertensive and nondiabetic Korean men. Metabolism 2008; 57: 569-76.

21. Targher G, Mantovani A, Pichiri I, et al. Nonalcoholic fatty liver disease is independently associated with an increased incidence of chronic kidney disease in patients with type 1 diabetes. Diabetes Care 2014; 37: 1729-36.

22. Burns RJ, Gibbons RJ, Yi Q, et al. The relationships of left ventricular ejection fraction, end-systolic volume index and infarct size to six-month mortality after hospital discharge following myocardial infarction treated by thrombolysis. J Am Coll Cardiol 2002; 39: 30-6.

23. Liu HL, Jin ZG, Yang SL, et al. Five-year outcomes of ST-elevation myocardial infarction versus non-ST-elevation acute coronary syndrome treated with biodegradable polymer-coated sirolimus-eluting stents: insights from the CREATE trial. J Cardiol 2017; 69: 149-55.

24. Heusch G. Reduction of infarct size by ischaemic postconditioning in humans: fact or fiction? Eur Heart J 2012; 33: 13-5.

25. Marenzi G, Moltrasio M, Assanelli E, et al. Impact of cardiac and renal dysfunction on inhospital morbidity and mortality of patients with acute myocardial infarction undergoing primary angioplasty. Am Heart J 2007; 153: 755-62.

26. De Luca G, Gibson CM, Gyöngyösi M, et al. Gender-related differences in outcome after ST-segment elevation myocardial infarction treated by primary angioplasty and glycoprotein IIb-IIla inhibitors: insights from the EGYPT cooperation. J Thromb Thrombolysis 2010; 30: 342-6.

27. Levine GN, Bates ER, Blankenship JC, et al. 2015 ACC/ AHA/SCAl Focused Update on Primary Percutaneous Coronary Intervention for Patients With ST-Elevation Myocardial Infarction: An Update of the 2011 ACCF/ AHA/SCAI Guideline for Percutaneous Coronary Intervention and the 2013 ACCF/AHA Guideline for the Management of ST-Elevation Myocardial Infarction: A Report of the American College of Cardiology/American Heart Association Task Force on Clinical Practice Guidelines and the Society for Cardiovascular Angiography and Interventions. Circulation 2016; 87: 1001-19.

28. Amsterdam EA, Wenger NK, Brindis RG, et al. 2014 AHA/ ACC guideline for the management of patients with non-ST-elevation acute coronary syndromes: a report of the American College of Cardiology/American Heart Association Task Force on Practice Guidelines. Circulation 2014; 130: e344-426.

29. Neumann U, Riemenschneider M, Sowa JP, et al. Compensation of feature selection biases accompanied with improved predictive performance for binary classification by using a novel ensemble feature selection approach. BioData Min 2016; 9: 36.

30. Dechêne A, Jochum C, Fingas C, et al. Endoscopic management is the treatment of choice for bile leaks after liver resection. Gastrointest Endosc 2014; 80: 626-33.e1.

31. Sowa JP, Heider D, Bechmann LP, et al. Novel algorithm for non-invasive assessment of fibrosis in NAFLD. PloS One 2013; 8: e62439.

32. Robin X, Turck N, Hainard A, et al. pROC: an open-source package for $\mathrm{R}$ and $\mathrm{S}+$ to analyze and compare $\mathrm{ROC}$ curves. BMC Bioinformatics 2011; 12: 77.

33. Guagliumi G, Stone GW, Cox DA, et al. Outcome in elderly patients undergoing primary coronary intervention for acute myocardial infarction: results from the Controlled Abciximab and Device Investigation to Lower Late Angioplasty Complications (CADILLAC) trial. Circulation 2004; 110: 1598-604.

34. Dunn W, Xu R, Wingard DL, et al. Suspected nonalcoholic fatty liver disease and mortality risk in a population-based cohort study. Am J Gastroenterol 2008; 103: 2263-71.

35. Barauskas M, Unikas R, Tamulenaite E, Unikaite R. The impact of clinical and angiographic factors on percutaneous coronary angioplasty outcomes in patients with acute ST-elevation myocardial infarction. Arch Med Sci Atheroscler Dis 2016; 2016: 150-7.

36. Giannitsis E, Lehrke S, Wiegand UK, et al. Risk stratification in patients with inferior acute myocardial infarction treated by percutaneous coronary interventions: the role of admission troponin T. Circulation 2000; 102: 2038-44.

37. Ferrer-Hita JJ, Dominguez-Rodriguez A, Garcia-Gonzalez MJ, Abreu-Gonzalez P. Renal dysfunction is an independent predictor of in-hospital mortality in patients with ST-segment elevation myocardial infarction treated with primary angioplasty. Int J Cardiol 2007; 118: 243-5.

38. Yamaguchi J, Kasanuki H, Ishii Y, et al. Serum creatinine on admission predicts long-term mortality in acute 
myocardial infarction patients undergoing successful primary angioplasty: data from the Heart Institute of Japan Acute Myocardial Infarction (HIJAMI) Registry. Circ J 2007; 71: 1354-9.

39. Halkin A, Singh M, Nikolsky E, et al. Prediction of mortality after primary percutaneous coronary intervention for acute myocardial infarction: the CADILLAC risk score. J Am Coll Cardiol 2005; 45: 1397-405.

40. Best PJM, Lennon R, Ting $\mathrm{HH}$, et al. The impact of renal insufficiency on clinical outcomes in patients undergoing percutaneous coronary interventions. J Am Coll Cardiol 2002; 39: 1113-9.

41. Karmen A, Wroblewski F, Ladue JS. Transaminase activity in human blood. J Clin Invest 1955; 34: 126-31.

42. Moon J, Kang W, Oh PC, et al. Serum transaminase determined in the emergency room predicts outcomes in patients with acute ST-segment elevation myocardial infarction who undergo primary percutaneous coronary intervention. Int J Cardiol 2014; 177: 442-7.

43. Lazzeri C, Valente S, Tarquini R, et al. Prognostic values of admission transaminases in ST-elevation myocardial infarction submitted to primary angioplasty. Med Sci Monit 2010; 16: CR567-574.

44. Yun KE, Shin CY, Yoon YS, Park HS. Elevated alanine aminotransferase levels predict mortality from cardiovascular disease and diabetes in Koreans. Atherosclerosis 2009; 205: 533-7.

45. Sowa JP, Atmaca Ö, Kahraman A, et al. Non-invasive separation of alcoholic and non-alcoholic liver disease with predictive modeling. PloS One 2014; 9: e101444.

46. Wree A, Kahraman A, Gerken G, Canbay A Obesity affects the liver - the link between adipocytes and hepatocytes. Digestion 2011; 83: 124-33.

47. Fracanzani AL, Burdick L, Raselli S, et al. Carotid artery intima-media thickness in nonalcoholic fatty liver disease. Am J Med 2008; 121: 72-8.

48. Targher G, Chonchol M, Bertolini L, et al. Increased risk of CKD among type 2 diabetics with nonalcoholic fatty liver disease. J Am Soc Nephrol 2008; 19: 1564-70.

49. Musso G, Gambino R, Tabibian JH, et al. Association of non-alcoholic fatty liver disease with chronic kidney disease: a systematic review and meta-analysis. PLoS Med 2014; 11: e1001680.

50. Ong JP, Pitts A, Younossi ZM. Increased overall mortality and liver-related mortality in non-alcoholic fatty liver disease. J Hepatol 2008; 49: 608-12.

51. Ekstedt $M$, Hagström $H$, Nasr P, et al. Fibrosis stage is the strongest predictor for disease-specific mortality in NAFLD after up to 33 years of follow-up. Hepatology 2015; 61: 1547-54.

52. Stepanova M, Younossi ZM. Independent association between nonalcoholic fatty liver disease and cardiovascular disease in the US population. Clin Gastroenterol Hepatol 2012; 10: 646-50.

53. Catalano D, Trovato GM, Martines GF, Pirri C, Trovato FM Renal function and severity of bright liver. Relationship with insulin resistance, intrarenal resistive index, and glomerular filtration rate. Hepatol Int 2011; 5: 822-9.

54. Targher G, Bertolini L, Rodella S, et al. Relationship between kidney function and liver histology in subjects with nonalcoholic steatohepatitis. Clin J Am Soc Nephrol 2010; 5: 2166-71

55. Kälsch J, Bechmann LP, Heider D, et al. Normal liver enzymes are correlated with severity of metabolic syndrome in a large population based cohort. Sci Rep 2015; 5: 13058 .
56. Kälsch J, Keskin H, Schütte A, et al. Patients with ultrasound diagnosis of hepatic steatosis are at high metabolic risk. Z Für Gastroenterol 2016; 54: 1312-9.

57. Littnerova S, Kala P, Jarkovsky J, et al. GRACE Score among six risk scoring systems (CADILLAC, PAMI, TIMI, Dynamic TIMI, Zwolle) demonstrated the best predictive value for prediction of long-term mortality in patients with ST-elevation myocardial infarction. PLoS One 2015; 10: e0123215.

58. Jelavic MM, Babic Z, Pintaric $H$. The importance of two metabolic syndrome diagnostic criteria and body fat distribution in predicting clinical severity and prognosis of acute myocardial infarction. Arch Med Sci 2016; 13: 795-806. 